\title{
UCRL-TR-223746
}

LA W RENCE LIVERMORE NATIONAL LABORATORY

\section{Hot dense matter creation in short-pulse laser interaction with tamped foils}

S.N. Chen, J.R. Pasley, F.N. Beg, G. Gregori, R. G. Evans, M. Notley, A.J. Mackinnon, S.H. Glenzer, S.B. Hansen, J.A. King, H.-K. Chung, S.C. Wilks, R.B. Stephens, R.R. Freeman, R.L. Weber, E. Garcia Saiz, F. Khattak, D. Riley

August 16, 2006 
This document was prepared as an account of work sponsored by an agency of the United States Government. Neither the United States Government nor the University of California nor any of their employees, makes any warranty, express or implied, or assumes any legal liability or responsibility for the accuracy, completeness, or usefulness of any information, apparatus, product, or process disclosed, or represents that its use would not infringe privately owned rights. Reference herein to any specific commercial product, process, or service by trade name, trademark, manufacturer, or otherwise, does not necessarily constitute or imply its endorsement, recommendation, or favoring by the United States Government or the University of California. The views and opinions of authors expressed herein do not necessarily state or reflect those of the United States Government or the University of California, and shall not be used for advertising or product endorsement purposes.

This work was performed under the auspices of the U.S. Department of Energy by University of California, Lawrence Livermore National Laboratory under Contract W-7405-Eng-48. 


\section{Hot dense matter creation in short-pulse laser interaction with tamped foils}

S. N. Chen, J. R. Pasley, F. N. Beg

University of California, San Diego, CA, 92093 USA

G. Gregori, R. G. Evans, M. Notley

CCLRC Rutherford Appleton Laboratory, Chilton, Oxfordshire, UK

S. H. Glenzer, S. B. Hansen, J. A. King, A. J. Mackinnon, H.-K. Chung, S. C. Wilks

Lawrence Livermore National Laboratory, Livermore, CA, 94551 USA

R. B. Stephens

General Atomics, San Diego, CA, 92121 USA

R. R. Freeman, R. L. Weber

The Ohio State University, Columbus, OH, 43210 USA

E. Garcia Saiz, F. Khattak, D. Riley

Queens University of Belfast, Belfast, BT7 1NN, N. Ireland, UK

Main contact email address: sophchen@ucsd.edu

Introduction

The possibility of producing hot dense matter has important applications for the understanding of transport processes in inertial confinement fusion (ICF) [1] and laboratory astrophysics experiments [2]. While the success of ICF requires the correct solution of a complex interaction between laser coupling, equation-of-state, and particle transport problems, the possibility of experimentally recreating conditions found during the ignition phase in a simplified geometry is extremely appealing.

In this paper we will show that hot dense plasma conditions found during ICF ignition experiments can be reproduced by illuminating a tamped foil with a high intensity laser. We will show that temperatures on the order of kiloelectronvolts at solid densities can be achieved under controlled conditions during the experiment. Hydrodynamic tamping by surface coatings allows to reach higher density regimes by enabling the diagnosis of matter that has not yet begun to decompress, thus opening the possibility of directly investigating strongly coupled systems [3]. Our experimental diagnostics is based on K-shell spectroscopy coupled to $\mathrm{x}$-ray imaging techniques. Such techniques have recently become prevalent in the diagnosis of hot dense matter [4]. By looking at the presence, and relative strengths, of lines associated with different ionization states, spectroscopy provides considerable insight into plasma conditions. At the same time, curved crystal imaging techniques allow for the spatial resolution of different regions of the target, both allowing for comparison of heating processes with the results of Particle-In-Cell (PIC) and hybrid simulation codes.

\section{Experiment}

The experiment described in this paper was carried out in Target Area West of the Vulcan laser facility at the Rutherford Appleton laboratory (UK). We have irradiated tamped solid foils, using the chirped pulse amplified (CPA) arm of the Vulcan laser delivering $\sim 85 \mathrm{~J}$ of $1.053 \mu \mathrm{m}$ light in either 1.5 or $10 \mathrm{ps}$ pulse length. The beam was focused down to a $10 \mu \mathrm{m}$ diameter spot by a $\mathrm{f} / 3$ parabola. Focused intensities were on the order of $3 \times 10^{19} \mathrm{~W} / \mathrm{cm}^{2}$. The targets (see Fig. 1), consisting of a square $5 \mu \mathrm{m}$ thick Ti sandwiched on each by either $1 \mu \mathrm{m} \mathrm{Al}$ or 2 $\mu \mathrm{m} \mathrm{CH}$, were mounted such that their normal was $30^{\circ}$ from the laser axis, to prevent back reflections onto the laser system. Two target sizes were used, $250 \times 250 \mu \mathrm{m}^{2}$ or $1 \times 1 \mathrm{~mm}^{2}$ squares. The targets were formed by coating the tamper onto the surface of the Titanium foil such that no glue was used between the layers.
A flat highly oriented pyrolytic graphite (HOPG) crystal [5] coupled to a FujiFilm image plate detector [6] viewed the back emission from the target in the $4-6 \mathrm{keV}$ region. A spherical quartz crystal, also coupled to an image plate detector, was used to spatially image the narrowband $4.51 \mathrm{~K}-\alpha \mathrm{Ti}$ emission [7] from the rear surface of the target.

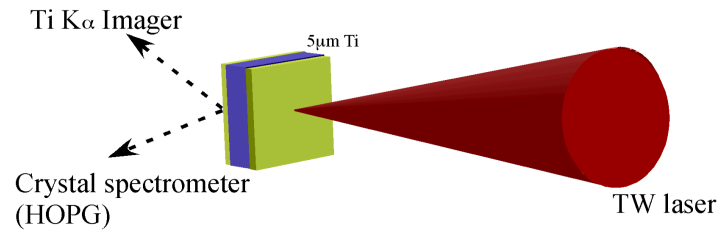

Figure 1: Experimental setup

\section{Results and Discussion}

The measured Ti K-shell emission from the Al-tamped target is shown in Fig. 2. The units of intensity have been linearized to units of PSL [8], where PSL represents radiation dose stored in the image plate. It is worth noting that the presence of He-like

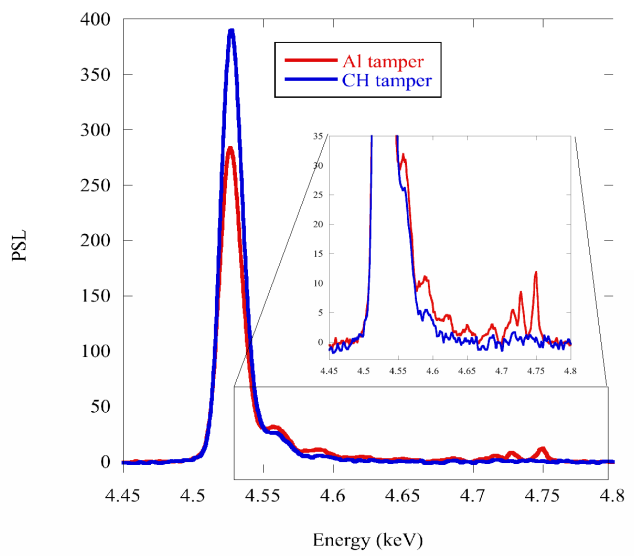

Figure 2: Experimental spectra showing various $K$-shell ionization states ( from cold $\mathrm{K \alpha}$ at $4.51 \mathrm{keV}$ to He-like 4.75 $\mathrm{keV})$ resulting from the target tamped with $1 \mu \mathrm{m} \mathrm{Al}$ and from the $2 \mu \mathrm{m}$ CH tamped target. Both targets were 250x250 $\mu^{2}$ in size. The laser pulse length was $1.5 \mathrm{ps}$. 
lines indicates temperatures $>300 \mathrm{eV}$.

Since the short pulse duration was much less than that over which significant hydrodynamic motion, changes in density during the interaction can be reasonably ignored. However, given the targets were only a few microns in thickness, the role of a pre-pulse (i.e., the presence of pedestal and nanosecond laser emission ahead of the primary pulse) in determining the target conditions was expected to be significant. The pre-pulse was measured to be $\sim 10^{6}$ times less intense than the primary pulse with a flat-top duration of $0.5 \mathrm{~ns}$.

The prepulse interacts with the $\mathrm{Al}$ or $\mathrm{CH}$ layer and launches a shock wave that propagates through the target thus causing the densities to rise above those for solid titanium (Fig. 3). The tamping layers were designed to limit expansion of the Titanium after the shock passes, but some hydrodynamic motion of this central region was expected. HELIOS [9], a 1-D radiationhydrodynamics code was used to estimate the conditions at the moment of the short pulse interaction. Fig. 3 shows that the bulk of the Titanium is compressed to approximately $6 \mathrm{~g} / \mathrm{cc}$.

(a)

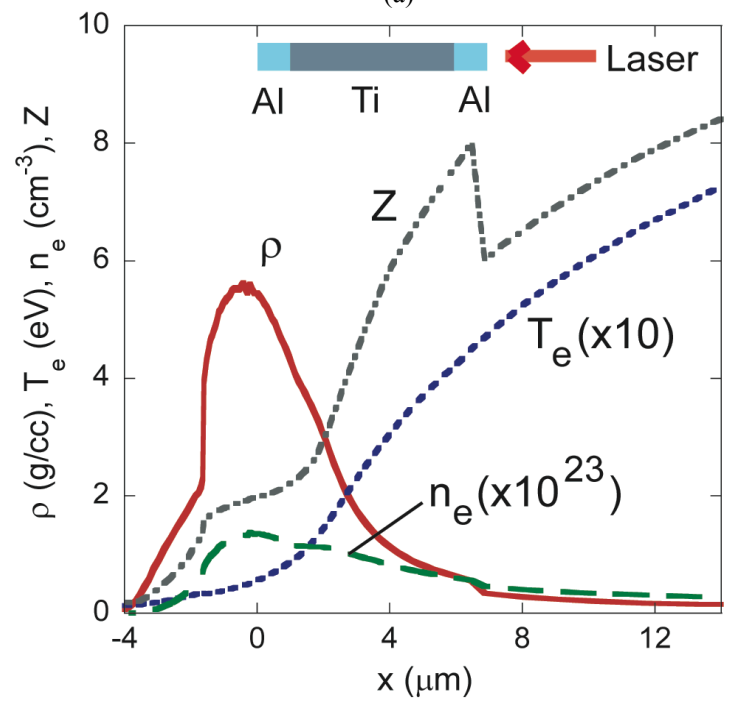

(b)

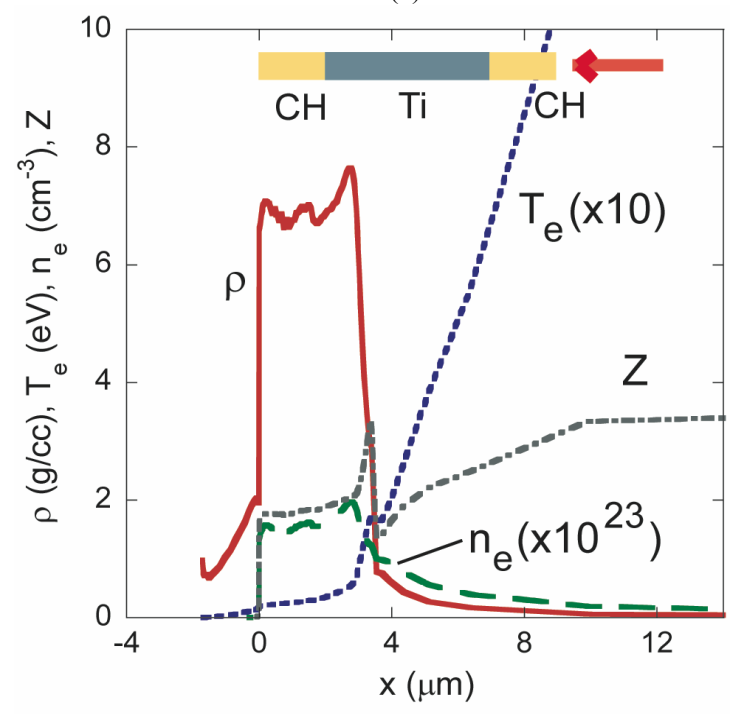

Figure 3: Simulation results of plasma condition of Al tamped target (a) and $\mathrm{CH}$ tamped target (b), arising from the 30 $T W / \mathrm{cm}^{2}$ pre-pulse just before the arrival of the main shortpulse.
When the main short-pulse arrives, hot electrons are generated at, or around, the critical surface. From the simulation we see that the critical surface is situated around $15 \mu \mathrm{m}$ away from the ablation front at the moment the peak of the pulse reaches the target. The beam of hot electrons produced by the short pulse is expected to be contained within a $15^{\circ}$ cone [10].

To estimate the temperatures to which the plasma was heated by the hot electrons, the experimental spectra have been compared to the predictions of the atomic physics package MuZe [3,11], showing that electron temperature in excess of $1 \mathrm{keV}$ are consistent with the appearance of the He- $\alpha$ line. Our spectra calculations have simplified the pre-plasma conditions by describing the target as two slabs of differing density - one to mimic the compressed / solid density region, and the other to the decompressed portion. The two densities employed were 6 and $2 \mathrm{~g} / \mathrm{cc}$ respectively. As shown in Fig. 2, the Ti He- $\alpha$ from the rear surface was observed only with $\mathrm{Al}$ tamper and none with the $\mathrm{CH}$ tamper. This suggests lower peak temperatures obtained with $\mathrm{CH}$ tamper, indicating a less efficient coupling of the electron beam onto the solid Ti. Due to the presence of the prepulse, the front, $\mathrm{Al}$ or $\mathrm{CH}$ layer is heated up to $20-60 \mathrm{eV}$ (see Fig. 2), thus destroying, when the main short-pulse arrives, any possible difference in hot electron transport due to conduction/valence band or solid state effects.

Spatially resolved $\mathrm{K}-\alpha$ images of both $\mathrm{Al}$ and $\mathrm{CH}$ tamped targets are shown in Fig. 4. Since the K- $\alpha$ emission is directly proportional to the hot electron number, these images are indicative of the hot electron spreading at the rear surface. The measurement indicates that in the case of $\mathrm{CH}$ tamped target the hot electrons distribute of a much larger volume, probably as a result of filamentation instabilities [12], and consequently the specific energy deposited is significantly lower. We argue that this could explain the missing $\mathrm{He}-\alpha$ emission from $\mathrm{CH}$ tamped targets. Comparisons with the predictions of PIC and hybrid codes will be essential to determine the physical mechanism of electron transport behind the observed differences in the electron spatial distribution at the rear of the target. Also, measurements taken with different target size $\left(1 \times 1 \mathrm{~mm}^{2}\right)$ showed that either 1 or $2 \mu \mathrm{m} \mathrm{Al}$ tamper does not affect the size of the hot spot.

There was, however, with both $\mathrm{Al}$ and $\mathrm{CH}$ tampers, Ti K-shell emission from various ionization states, appearing as side peaks blue shifted from the primary cold $\mathrm{K}-\alpha$ line. This thus indicates that the temperature within the target varied considerably. The comparison done with the MuZe code suggests average temperature $\sim 125 \mathrm{eV}$, in agreement with previous work [3].

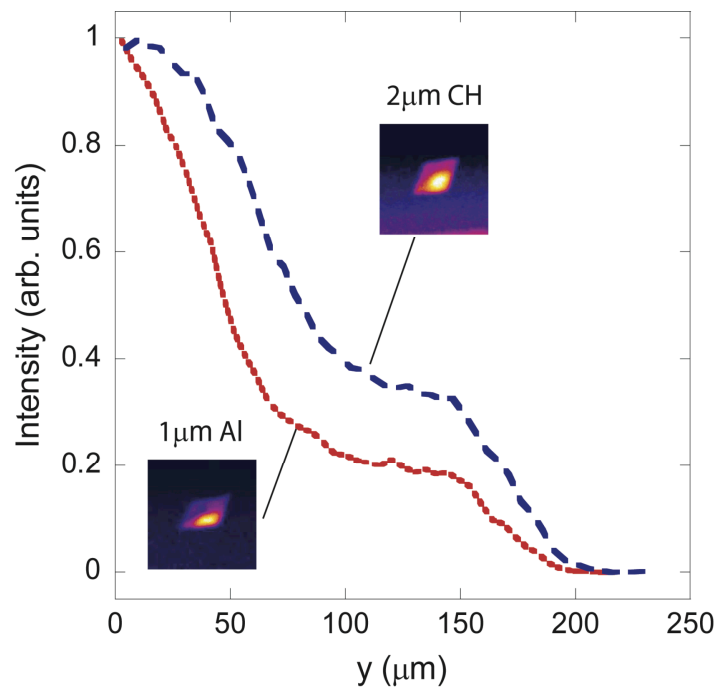

Figure 4: Measured spatial distribution of the K- $\alpha$ emission from the rear of $250 \times 250 \mu^{2}$ targets. The line profiles correspond to the spatial distribution of the hot spot intensity. The laser pulse length was $1.5 \mathrm{ps}$. 
Summary

We have successfully created a dense Ti plasma, with density $\sim 6 \mathrm{~g} / \mathrm{cc}$ and temperature above $1 \mathrm{keV}$. These conditions can be used to develop a test bed platform to study EOS and transport problems that are relevant to both ICF and laboratory astrophysics experiments.

Acknowledgements

This work was partially supported by the Council for the Central Laboratory of the Research Councils (UK) and S. N. C. was supported by Campus Laboratory Collaboration Program administrated by the President of the University of California (USA).

\section{References}

[1] J. D. Lindl, Inertial Confinement Fusion (Springer-Verlag, New York, 1998)

[2] B. A. Remington et al., Rev. Mod. Phys., 78, 755 (2006)

[3] G. Gregori et al., Contrib Plasma Phys., 45, 284 (2005)

[4] A. Saemann et al., Phys. Rev. Lett. 82, 4843 (1999)

[5] A. Pak et al., Rev. Sci. Instrum., 75, 374 (2004)

[6] J. Miyahara et al., Nucl. Instrum. Methods Phys. Res., A 246, 572 (1986)

[7] J. King et al., Rev. Sci. Instrum., 76, 6102 (2005)

[8] FujiFilm Corp. internal report

[9] Software from Prism Computational Science (www.prismcs.com)

[10] M.I.K Santala et al., Phys Rev. Lett. 84, 1459 (2000)

[11] S. B. Hansen et al., Phys. Rev. E, 72, 036408 (2005)

[12] P. A. Norreys et al., Plasma Phys. Controlled Fusion, 48,

L11 (2006) 